\title{
Numerical and experimental study of suffosion/clogging in deformable porous media
}

\author{
Maxim Khramchenkov ${ }^{1,2, *}$, Eduard Khramchenkov ${ }^{1,2}$, and Anastasiya Garaeva ${ }^{2}$ \\ ${ }^{1}$ NIISI RAS, Kazan branch, 420111, Kazan, Russia \\ ${ }^{2}$ Kazan Federal University, 420018, Kazan, Russia
}

\begin{abstract}
Mathematical model and experiment on suffosion/clogging process are presented. The model is based on the theory of flow in deformable porous media with mass-variable skeleton. It is shown that clogging occurs at a distance from injecting borehole. It is caused by precipitation of particles which were eroded by grounwater flow during the suffosion of the solid skeleton near the borehole.
\end{abstract}

\section{Suffosion and clogging}

During the oilfield development water injection is performed to maintain reservoir pressure. Distribution of pressure and flow velocity around a well has logarithmic character with highest values near the well [1]. A rate of water injection is dictated by strategy of reservoir development. If pressure gradient is sufficiently high then flow in pores can tear particles from a solid skeleton in some neighborhood of the well [2]. As a result void fraction of the porous medium grows, therefore porosity and permeability increase. So one can say there is an erosion process going in the neighborhood of the well. At a distance from the well where the flow rate drops, erosion declines and gives way to deposition of solid particles which were tore by the flow. Precipitating particles cause clogging of the pores and hence permeability decreases and it results in degradation of the reservoir [3]. In [4] authors show that deposition of eroded particles happens only when hydraulic gradient falls below some critical value. Clogging that is caused by deposition of solid particles in porous media is described by classic deep bed filtration models based on the conception of suspended and deposited particles [5,6]. We propose mathematical model for coupled suffosion/clogging process. Our model is based on the theory of mass-transfer in deformable porous medium with mass-variable skeleton. Main basis of this theory is presented in $[7,8]$. The advantage of our approach is that we don't need any presumptions and empiric statements to describe the process. We only need to specify the source term of the general model in order to use it for modelling of suffosion/clogging process.

\footnotetext{
* Corresponding author: mkhramch@gmail.com
} 


\section{Mathematical model}

According to [1] mass balance of dissolving fluid (water) and dissoluble solid skeleton can be described by the equation

$$
\frac{\partial \theta}{\partial t}+\operatorname{divq}=\frac{1-\phi}{V_{s}} \frac{\partial V_{s}}{\partial t}
$$

Here $\theta$ stands for dilatation, $\phi$ is porosity, $\mathbf{q}$ is a vector of flow velocity, $V_{s}$ stands for volume of solid phase of porous medium. Concentration of weighed solid particles in water is designated as $c$.

On the basis of the equation of mass transfer of material of solid phase from $[7,8]$ we have for $c$

$$
\frac{\partial(c \phi)}{\partial t}+\operatorname{div}(c \mathbf{q})+m c \frac{\partial \theta}{\partial t}=-\frac{1-\phi}{V_{s}} \frac{\partial V_{s}}{\partial t} \rho_{s}
$$

where $\rho_{s}$ is a density of a solid phase.

Rate of mass change of the solid phase caused by erosion and precipitation of particles is equal to the amount of particles in the volume that were tore and precipitated per unit of time. The velocity of suffosion and precipitation is determined by [9]. The rate of change of volume $V_{s}$ of the solid phase during the suffosion and clogging can be written as

$$
\beta c q\left(1+b\left(\phi_{0}-\phi\right)\right)-\alpha\left(\frac{q}{\phi}-q_{0}\right)\left(\phi-\phi_{0}\right) H\left((q / \phi)-q_{0}\right)=\frac{1-\phi}{V_{s}} \frac{\partial V_{s}}{\partial t} \rho_{s}
$$

where $q=|\mathbf{q}|, \alpha$ and $\beta$ are the constants of suffosion and clogging velocity correspondingly, in some situations $b$ was taken as 0 , while in other cases, $\mathrm{b}$ was taken as 1 [9]. Then the simulation of the process of water injection into the layer with constant discharge $Q$ was performed. The velocity of flow is

$$
q=\frac{Q}{2 \pi r L}
$$

where $r$ is distance from the borehole, $L$ is the thickness of the layer.

\section{Experiment on suffosion-deposition}

Samples from the Ufa terrigenous bitumen complex of Vishnevo-Polyanskoe oilfield, Severo-Karmalinskyoe oilfield and Kamenskoe oilfield are examined. The scheme of experimental setup is presented on Fig. 1. 


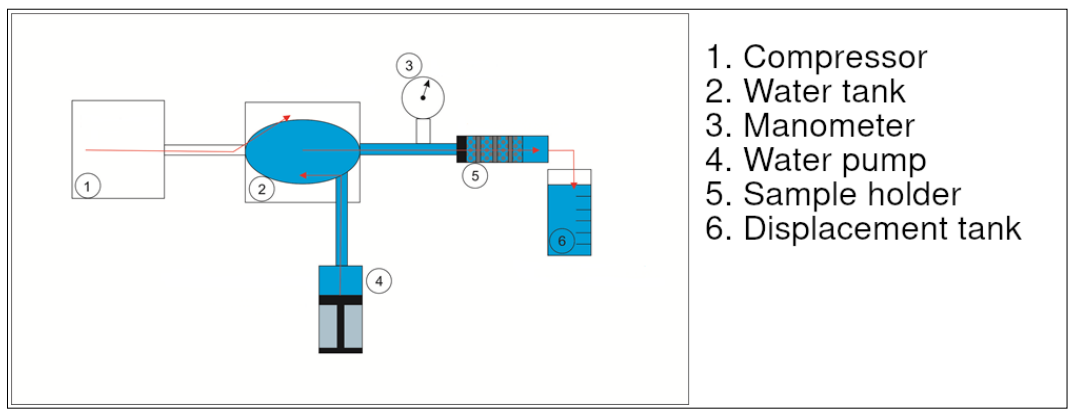

Fig. 1. Experimental setup.

The experiment was performed in three stages under constant thermobaric conditions.

\subsection{Stage 1. Clogging of a porous media and determination of its permeability}

After study of physical properties samples with similar permeability and mineralogical properties were selected. Properties were examined under constant pressure by filtration of distilled water through samples until stationary regime. Characteristics of samples are presented in Table 1.

Table 1. Samples characteristics.

\begin{tabular}{|c|c|c|c|c|c|c|}
\hline $\begin{array}{c}\text { № } \\
\text { sample }\end{array}$ & Age & $\begin{array}{c}\text { Depth, } \\
\text { m. }\end{array}$ & $\begin{array}{c}\text { Porosity, \% } \\
\text { (gas } \\
\text { permeameter) }\end{array}$ & $\begin{array}{c}\text { Water } \\
\text { permeability, } \\
\text { under } 1 \\
\text { atm., }{ }^{*} 10^{-3} \mu^{2}\end{array}$ & $\begin{array}{c}\text { Permeability } \\
\text { after } \\
\text { colmatation } \\
\text { under } 1 \\
\text { atm., }{ }^{*} 10^{-3} \mu \mathrm{m}^{2} \\
\end{array}$ & $\begin{array}{c}\text { Permeability } \\
\text { after } \\
\text { colmatation } \\
\text { under } 1 \\
\text { atm. }{ }^{*} 10^{-3} \mu \mathrm{m}^{2}\end{array}$ \\
\hline $24 / 1$ & Piuf $_{2}$ & 105,5 & 24,32 & 9,66 & 1,50 & 1,78 \\
\hline $24 / 2$ & $\mathrm{P}_{1} \mathrm{uf}_{2}$ & 105,5 & 23,68 & 8,51 & 1,78 & 2,75 \\
\hline $24 / 158 / 2$ & $\mathrm{P}_{1} \mathrm{uf}_{2}$ & 158 & 27,44 & 8,41 & 1,28 & 1,44 \\
\hline $33 / 3$ & $\mathrm{P}_{1} \mathrm{f}_{2}$ & 144,1 & 22 & 8,42 & 0,96 & 1,34 \\
\hline $33 / 1$ & Piuf $_{2}$ & 144,1 & 22,52 & 7,45 & 0,49 & 1,10 \\
\hline $33 / 2$ & $\mathrm{P}_{1} \mathrm{uf}_{2}$ & 144,1 & 22,15 & 7,85 & 0,51 & 0,72 \\
\hline $17 / 1$ & $\mathrm{P}_{1} \mathrm{uf}_{2}$ & 163,5 & 22,05 & 9,40 & 2,48 & 2,70 \\
\hline $17 / 2$ & Piuf $_{2}$ & 163,5 & 22,63 & 9,40 & 2,38 & 2,55 \\
\hline $20 / 1$ & $\mathrm{P}_{1} \mathrm{uf}_{2}$ & 131,5 & 26,7 & 8,40 & 2,38 & 2,62 \\
\hline $20 / 2$ & $\mathrm{P}_{1} \mathrm{uf}_{2}$ & 131,5 & 26,94 & 10,20 & 2,10 & 2,60 \\
\hline $21 / 2$ & $\mathrm{P}_{1} \mathrm{uf}_{2}$ & 148,1 & 16,69 & 6,72 & 1,19 & 2,75 \\
\hline $21 / 3$ & $\mathrm{P}_{1} \mathrm{uf}_{2}$ & 148,1 & 16,2 & 6,90 & 1,20 & 1,34 \\
\hline $21 / 1$ & $\mathrm{P}_{1} \mathrm{ff}_{2}$ & 148,1 & 17,22 & 6,82 & 1,50 & 1,46 \\
\hline $12 / 1$ & $\mathrm{P}_{1} \mathrm{uf}_{2}$ & 143 & 15,2 & 2,29 & 0,70 & 0,62 \\
\hline
\end{tabular}




\subsection{Stage 2. Colmatation modeling}

Solution of bentonite from Biklyanskoe oilfield (Tatarstan, Russia) was used as a filtrating fluid. Clay component was determined by hydrometer test with segregation of clay particles less than $5 \mu \mathrm{m}$. According to all-Union State Standard 12536-7 measurements of solution density were conducted during sedimentation of clayey suspension. Density of clay solution was measured before and after the experiment. Filtration of the solution was conducted until stationary regime. Due to contamination of a porous medium sharp degradation of permeability of samples was observed.

\subsection{Stage 3. Examination of permeability of clogged samples}

Clogged porous samples were examined. Permeability was examined under constant pressure by filtration of distilled water through samples until stationary regime. As a result increase of permeability in all samples was detected caused by disengagement of clay particles deposited during the Stage 2. It should be noted that process has a wave-like character. It is connected with secondary deposition of disengaged particles and causing subsequent reduction of permeability. Fig. 2 demonstrates that these oscillations disappear and permeability becomes stationary. It should be noted that final permeability of the samples is higher (by 10\%-50\%) than permeability after Stage 2 . It shows that share of clay particles were washed out from the samples that is confirmed by measurement of mass of the filtrated fluid.

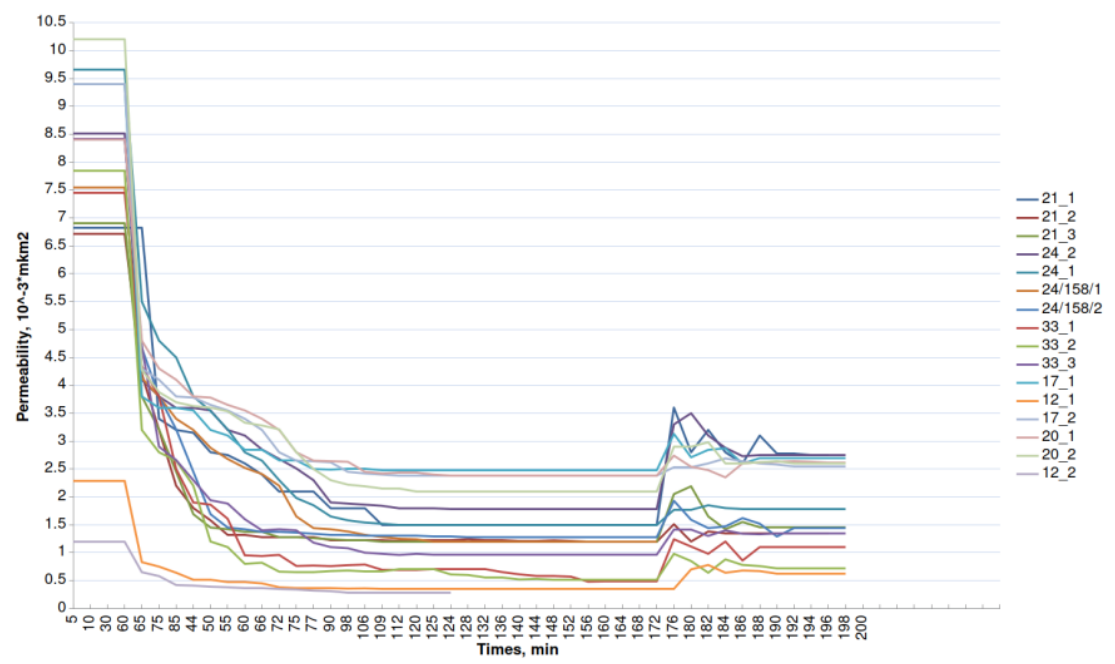

Fig. 2. Permeability of samples during 3 stages of the experiment.

\section{Numerical solution}

Simplified 1-D model was solved first to simulate the performed experiment. For flow velocity 1-D Darcy law was used. Tuning parameters of the model were fitted on the basis of the experimental data. Averaged values of porosity and permeability of the samples were used as an initial parameters for the numerical model. All constant values were taken from the experiment. Stages of the experiment were reproduced in numerical simulations. Significant dependence of porosity distribution on the values of the parameters was demonstrated. Result of the numerical test is presented on Fig. 3. Close fit of solution profile with experimental results is achieved. 


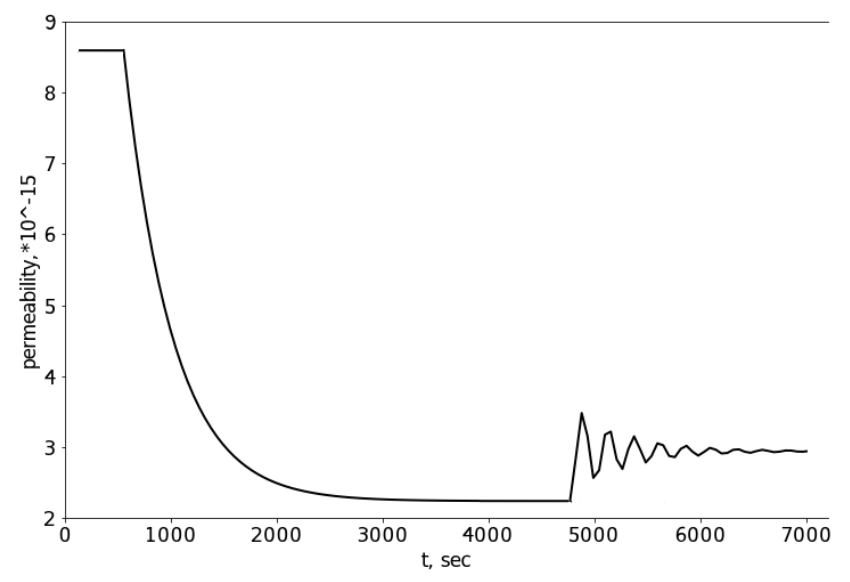

Fig. 3. One-dimensional numerical test.

\section{Conclusions}

Experimental and numerical investigation of erosion-deposition in deformable porous media was performed. The mathematical model of the process is developed. Simplified 1D test model was solved to verify mathematical model and to estimate tuning parameters. The solution of the simplified model shows good correspondence with experimental results. Both experimental and numerical results revealed wave-like behaviour of the process which is untypical for the flow in porous media. Wave-like behaviour is caused by intersperse of two concurrent processes: erosion of porous channels and clogging of pores by suspended particles.

\section{References}

1. A. Tarek, P. McKinney, Advanced reservoir engineering (Elsevier, 2011)

2. A.Kudrolli, X. Clotet, Phys. Rev. Lett., 117 (2), 028001 (2016)

3. A. Bouddour, J.L. Auriault, M. Mhamdi-Alaoui, TRANSPORT POROUS MED, 25 (2), 121-146 (1996)

4. A. Chetti, A. Benamar, A. Hazzab, TRANSPORT POROUS MED, 113 (3), 591-606 (2016)

5. P. Herzig, D.M. Leclerc, P. Le Goff, Industrial \& Engineering Chemistry, 62 (5), 8-35, (1970)

6. T. Iwasaki, J.J. Slade, Wm. E. Stanley, Journal (American Water Works Association), 29(10), 1591-1602 (1937)

7. M. Khramchenkov, E. Khramchenkov, Int. J. Rock Mech. Min. Sci., 72, 49-53 (2014)

8. M. Khramchenkov, E. Khramchenkov, Int. J. Rock Mech. Min. Sci., 86, 1-4 (2016)

9. E.S. Boek, C. Hall, P. Tardy, TRANSPORT POROUS MED, 91 (2), 479-508 (2012) 